\title{
Ionizing radiation induced emission: scintillation and storage-type luminescence
}

Takayuki Yanagidaa, $\left.{ }^{*}\right)$

a Kyushu Institute of Technology, 2-4 Hibikino, Wakamatsu, Kitakyushu, Fukuoka, 808-0196, Japan

\begin{abstract}
In solid state ionizing radiation detectors, mainly two types of luminescent materials, scintillators and dosimeter materials, have been investigated for a long time. The former one transduces the energy of high energy ionizing radiation $(\mathrm{keV}-\mathrm{GeV})$ to thousands of UV-visible photons immediately and the latter storages energies under radiation exposure. After the excitation by visible photons (OSL) or thermal energies (TSL), dosimeter materials release the storage energies by UV-visible emission. In this work, Ce-doped $\mathrm{CaF}_{2}$, Eu-doped $\mathrm{LiCaAlF}_{6}, \mathrm{Ce}$-doped $\mathrm{LiCaAlF}_{6}$, and Pr-doped LuAG were evaluated on their scintillation, OSL, and TSL properties. In Ce-doped $\mathrm{CaF}_{2}$, Eu-doped $\mathrm{LiCaAlF}_{6}$, and Pr-doped LuAG, scintillation and dosimeter properties showed a complementally relation while Ce-doped $\mathrm{LiCaAlF}_{6}$ exhibited different properties.
\end{abstract}

Keywords: $\quad$ Scintillator; OSL; TSL; $\mathrm{CaF}_{2} ; \mathrm{LiCaAlF}_{6} ; \mathrm{LuAG}$

${ }^{*}$ Corresponding author. Present address: Kyushu Institute of Technology, 2-4 Hibikino, Wakamatsu, Kitakyushu, Fukuoka, 808-0196, Japan

Tel.: +8193695 6049; fax: +81936956049.

E-mail address: yanagida@1sse.kyutech.ac.jp (Takayuki Yanagida)

(C) 2015. This manuscript version is made available under the Elsevier user license http://www.elsevier.com/open-access/userlicense/1.0/ 


\section{Introduction}

In solid state ionizing radiation detectors, mainly two types of luminescent materials, scintillators and dosimeter materials, have been investigated for a long time [1-2]. The former one transduces the absorbed energy of high energy ionizing radiation ( $\mathrm{keV}-\mathrm{GeV})$ to thousands of $\mathrm{UV}$-visible photons immediately (scintillation) [3] and the latter storages energies under radiation exposure. After the excitation by visible photons (optically stimulated luminescence; OSL) [4] or thermal energies (thermally stimulated luminescence; TSL) [5], dosimeter materials release the storage energies by UV-visible emission. Although basic processes, absorption of the energy of ionizing radiation and release of absorbed energies by luminescence, are the same, no one treat these phenomena uniformly due to a huge discrepancy of measurement techniques in scintillators and dosimeters. Recently, we proposed a hypothesis that scintillation and dosimeter-type luminescence properties should be complementary assuming the energy conservation. If some materials are not bright in scintillation, they will storage some portions of absorbed energies and show high luminosity as dosimeters, and vice versa. Recently, this hypothesis was confirmed experimentally in Ce differently doped $\mathrm{CaF}_{2}$ crystals [6].

To investigate further about ionizing radiation induced luminescence, in this time, I evaluated scintillation, OSL, and TSL properties of Eu-doped (1, 2, 3, and 4\%) $\mathrm{LiCaAlF}_{6}[7-8]$, Ce-doped (2, 3, and 4\%) LiCaAlF 6 [9-10], and Pr-doped $(0.25,0.3,0.4,0.6,0.8$, and $1 \%) \mathrm{LuAG}[11-12]$ on a comparison with previously studied Ce-doped $\mathrm{CaF}_{2}[6]$. $\mathrm{LiCaAlF}_{6}$ crystals were fabricated by Tokuyama Corp. and these materials became commercially available products. They had a size of $10 \times 10 \times 1 \mathrm{~mm}^{3}$. Transparent ceramic Pr-doped LuAG and crystalline counterpart were prepared by Konoshima Chemical and Furukawa, respectively. Sample size of Pr-doped LuAG was $5 \times 5 \times 1 \mathrm{~mm}^{3}$. Up to now, dosimeter properties of $\mathrm{Eu}$ or Ce-doped $\mathrm{LiCaAlF}_{6}$ were sometimes reported independently [13-14] and those for Pr-doped LuAG were for the first time. In Ce-doped $\mathrm{LiCaAlF}_{6}$, I also examined both micro-pulling down $(1,3$, and 5\%) and conventional Czochralski method grown crystals to study any effects on dosimeter properties by fabrication methodologies. Typical size of micro-pulling down samples was $2 \times 5-7 \times 1 \mathrm{~mm}^{3}$.

\section{Experimental procedures}

As mentioned above, sample sizes were few $\mathrm{mm}^{2}$ to $1 \mathrm{~cm}^{2}$ with a thickness of $1 \mathrm{~mm}$. At least in the same material system, sample sizes were arranged similar. At first, basic scintillation responses including radioluminescence, 
scintillation decay, and scintillation light yield were evaluated to confirm that present samples were not special ones. X-ray induced radioluminescence spectra were evaluated and experimental geometry was described previously [15]. The source was X-ray generator supplied with $40 \mathrm{kV}$ bias voltage and $5.2 \mathrm{~mA}$ tube current. Scintillation decay time profiles were evaluated by using pulse X-ray equipped afterglow characterization system in the fast mode [16]. In this system, wavelength was not resolved and photons from 160 to $650 \mathrm{~nm}$ were accumulated. Scintillation light yield under ${ }^{241} \mathrm{Am} \alpha$-ray for Ce-doped $\mathrm{CaF}_{2},{ }^{252} \mathrm{Cf}$ neutron for $\mathrm{Ce}$ and Eu-doped $\mathrm{LiCaAlF}_{6}$, and ${ }^{137} \mathrm{Cs} \gamma$-ray for Pr-doped LuAG, were investigated. I used PMT R7600-200 and typical experimental setup was published previously [17].

In OSL and TSL properties, X-ray generator was used as an irradiation source. The bias voltage and tube current were same with those in X-ray induced radioluminescence, $40 \mathrm{kV}$ and $5.2 \mathrm{~mA}$, respectively. The irradiated dose to samples was calibrated by using an ionization chamber. OSL was evaluated by using Xe-lamp (Hamamatsu Quantaurus-QY) as a stimulation source for Ce-doped $\mathrm{CaF}_{2}$ after $1 \mathrm{~Gy}$ exposure and LED for other materials after 10 Gy exposure. Stimulation wavelengths were $550 \mathrm{~nm}$ for Ce-doped $\mathrm{CaF}_{2}, 470 \mathrm{~nm}$ for Eu-doped $\mathrm{LiCaAlF}_{6}, 405 \mathrm{~nm}$ for Ce-doped $\mathrm{LiCaAlF}_{6}$, and also $405 \mathrm{~nm}$ for Pr-doped LuAG. The OSL intensity of Ce-doped $\mathrm{CaF}_{2}$ was enough strong so that the emission intensity could be evaluated quantitatively by photoluminescence quantum yield. On the other hand, OSL of other materials were not so strong and they were stimulated by LED which was stable and high-power than Xe-lamp. In TSL, exposure dose to Eu- and Ce-doped LiCaAlF $_{6}$ was 1 Gy and that to Pr-doped LuAG was 10 mGy because TSL sensitivity of Pr-doped LuAG was so strong. I could not evaluate TSL of Ce-doped $\mathrm{CaF}_{2}$ since some of the samples broke through temperature change. TSL was evaluated by using TL2000 (nanogray) [18] and the heating rate was $1{ }^{\circ} \mathrm{C} / \mathrm{s}$. TSL intensity was evaluated by sum of all signals from 50 to $450{ }^{\circ} \mathrm{C}$.

Finally, scintillation light yield and OSL or TSL intensities were plotted. Unfortunately, evaluation methodology of quantitative values for dosimeter properties have not been established yet and I could not compare absolute dosimeter intensities of samples with different chemical compositions. One of the idea to evaluate OSL intensity is photoluminescence quantum yield but the weak point of this technique is a request for bright OSL materials. Generally, evaluation systems for photoluminescence quantum yield are designed for bright materials (e.g., display material) and they do not suit to investigate faint light. To develop novel new methodologies for quantitative evaluations for dosimeter materials is a problem to be solved. 


\section{Experimental results and discussion}

\subsection{Scintillation properties}

X-ray induced radioluminescence spectra of Eu-doped $\mathrm{LiCaAlF}_{6}, \mathrm{Ce}$-doped $\mathrm{LiCaAlF}_{6}$, and selected samples of Pr-doped LuAG are presented in figure $1 . \mathrm{Eu}^{2+} 5 \mathrm{~d}-4 \mathrm{f}$ emission around $370 \mathrm{~nm}$ and weak $4 \mathrm{f}-4 \mathrm{f}$ emission around $580 \mathrm{~nm}$ appeared in $\mathrm{LiCaAlF}_{6}$ host. Then in Ce-doped $\mathrm{LiCaAlF}_{6}, \mathrm{Ce}^{3+} 5 \mathrm{~d}-4 \mathrm{f}$ emission was observed around $300 \mathrm{~nm}$. In Pr-doped LuAG, Pr 0.25\% doped crystal and ceramic were compared and $\operatorname{Pr}^{3+} 5 \mathrm{~d}-4 \mathrm{f}$ emission was detected around $310 \mathrm{~nm}$ and some $4 \mathrm{f}-4 \mathrm{f}$ lines were observed from 500 to $700 \mathrm{~nm}$. Observed scintillation spectra were typical in these materials. Scintillation spectra of Ce-doped $\mathrm{CaF}_{2}$ was published previously [6] and spectral shape was similar to Ce-doped $\mathrm{LiCaAlF}_{6}$.

Figure 2 represents scintillation decay time profiles of Eu-doped $\mathrm{LiCaAlF}_{6}, \mathrm{Ce}$-doped $\mathrm{LiCaAlF}_{6}$, and $\operatorname{Pr} 0.25 \%$ doped ceramic and crystal LuAG. Primary decay time of Eu-doped LiCaAlF 6 were 1.6-1.8 $\mu$ s and decay times monotonically fastened with Eu concentrations. In Ce-doped $\mathrm{LiCaAlF}_{6}$, primary decay times were $30-40 \mathrm{~ns}$ and these values were typical in Ce-doped fluoride scintillators. Scintillation decay time of Pr-doped LuAG was around 20 ns due to $\mathrm{Pr}^{3+} 5 \mathrm{~d}-4 \mathrm{f}$ transition. As same as $\mathrm{X}$-ray induced radioluminescence, observed scintillation decay time profiles were typical for these materials.

Figure 3 demonstrates ${ }^{252} \mathrm{Cf}$ induced newton induced pulse height spectra of Eu- and Ce-doped $\mathrm{LiCaAlF}_{6}$ and ${ }^{137} \mathrm{Cs}$ $\gamma$-ray induced pulse height of Pr $0.25 \%$ doped ceramic and crystal LuAG. In LiCaAlF 6 scintillators, clear thermal neutron peak appeared and scintillation light yield became larger higher dopant concentrations. Results on Pr-doped LuAG were basically similar ones since used samples and photodetectors were same with the previous report [12]. Clear photoabsorption and Lu escape peaks were observed in Pr-doped LuAG samples. Though I could deduce the absolute light yield of $\mathrm{LiCaAlF}_{6}$ scintillators, I used a raw signal intensity, MCA channel since OSL and TSL intensities could not be evaluated in absolute values.

\subsection{OSL and TSL properties}

OSL spectra of Eu-doped $\mathrm{LiCaAlF}_{6}, \mathrm{Ce}$-doped $\mathrm{LiCaAlF}_{6}$, and selected samples of Pr-doped LuAG are depicted in 
figure 4. OSL of Eu-doped $\mathrm{LiCaAlF}_{6}$ appeared around $370 \mathrm{~nm}$ and it was same with the scintillation shown in figure 1. In $\mathrm{Ce}$-doped $\mathrm{LiCaAlF}_{6}$, interesting phenomenon was observed. The dominant OSL wavelength of $\mathrm{Ce} 2 \%$ doped sample was $270 \mathrm{~nm}$ and $320 \mathrm{~nm}$ for others. In figure 1, scintillation peaks were mainly 270, $320 \mathrm{~nm}$, and 330-360 nm

(not resolved clearly) so that the origin of these OSL were $\mathrm{Ce}^{3+} 5 \mathrm{~d}-4 \mathrm{f}$ transition. In the inset of the panel of Ce-doped $\mathrm{LiCaAlF}_{6}$, results of micro-pulling down made crystals are also presented. Though fabrication techniques were different, similar results were observed. In Ce-doped $\mathrm{LiCaAlF}_{6}$, stimulation efficiency or release routes of trapped carriers would depends on dopant concentrations. Dominant OSL wavelength of Pr-doped LuAG was $310 \mathrm{~nm}$ and it was same with the scintillation. The secondary peak around $370 \mathrm{~nm}$ was not efficient.

TSL glow curves of Eu-doped $\mathrm{LiCaAlF}_{6}$, Ce-doped $\mathrm{LiCaAlF}_{6}$, and selected samples of Pr-doped LuAG are presented in figure 5. Glow peaks of Eu- and Ce-doped $\mathrm{LiCaAlF}_{6}$ were same with those in previous report [14] and very complicated features were observed. In Pr-doped LuAG, TSL sensitivity of this material was so high so that we could not evaluate TSL signals at high temperature range due to a saturation (detection limit). Lower temperature glow peak around $80^{\circ} \mathrm{C}$ was not so sensitive and I used this peak for this study. To my experience, radiation-weak materials show high OSL and TSL intensity. Recently, radiation tolerance of Pr-doped LuAG was investigated and this material was relatively weak to high dose radiation exposure [19-20]. Though TSL and OSL are qualitative evaluations, OSL and TSL intensity of Pr-doped LuAG were higher than other famous scintillators at least in my experience.

\subsection{Relation of scintillation and dosimeter properties}

Figure 6 exemplifies a relation between scintillation light yield (ph/5.5 MeV- $\alpha$ ) under ${ }^{241} \mathrm{Am} \alpha$-ray excitation and OSL (photoluminescence quantum yield) from some selected data of our recent work [6]. Clear reverse proportionality was observed in $\mathrm{Ce}$-doped $\mathrm{CaF}_{2}$ crystal system. The aim of this work is to examine similar experiments to other material systems, like other dopants, other anions, and material forms (crystal or ceramic).

Figure 7 represents the relation between the scintillation and OSL in Eu-doped $\mathrm{LiCaAlF}_{6}, \mathrm{Ce}-$ doped $\mathrm{LiCaAlF}_{6}$, and Pr-doped LuAG systems. In Eu-doped $\mathrm{LiCaAlF}_{6}$, similar relation with the case of Ce-doped $\mathrm{CaF}_{2}$ was observed and the complementary relation was confirmed in this material system. On the other hand, Ce-doped $\mathrm{LiCaAlF}_{6}$ exhibited different relation. Scintillation light yield and OSL intensity showed a positive proportionality. To investigate this 
tendency was affected by some special reasons like sample preparation status or not, micro-pulling down grown samples were evaluated in the same manner. As a result, the tendency was same and positive proportionality of scintillation and dosimeter intensity was a material property of Ce-doped $\mathrm{LiCaAlF}_{6}$ system. There would be some possibilities for interpretation. One is that some thresholds exist to satisfy the complementary relation, e.g., dopant or defect (trap site) concentrations. Different OSL spectra of Ce low and high concentration samples suggested that there would be some kinds of trap sites associated with kinds of Ce emission centers. Such a complicated crystalline field may affect the relation. In Pr-doped LuAG, the complementary relation was observed with some thresholds and OSL signal saturated at some levels. This saturation can be simply interpreted that all trap sites are fulfilled.

Figure 8 shows a same relation but for TSL. In all sample systems, tendencies were same with the relation between scintillation and OSL. Eu-doped $\mathrm{LiCaAlF}_{6}$ exhibited a clear reverse proportionality against scintillation while that of Ce-doped $\mathrm{LiCaAlF}_{6}$ showed a positive proportionality. Different point was that there was no saturation in Pr-doped LuAG. However, Pr-doped LuAG had a high TSL sensitivity and already saturated in high temperature range in figure 5 even in very low 10 mGy exposure. If I could evaluate TSL signal at lower dose and taking into consideration whole TSL signal, TSL signal may saturate as same as the case of OSL. In my system, the lower limit of the exposure was around $1 \mathrm{mGy}$ and it was technically difficult to calibrate such a low dose correctly in laboratory level. In addition, TSL was proportional to OSL in these Eu-doped $\mathrm{LiCaAlF}_{6}$, Ce-doped $\mathrm{LiCaAlF}_{6}$, and Pr-doped LuAG systems.

Up to now, the conventional understanding about scintillation is that few $\%$ of the absorbed energy is converted to scintillation photons and remaining energies becomes thermal loss. The present work at least points out that some portions of the absorbed energy is storage and in some material system scintillation and dosimeter properties exhibit inverse proportionality. It is a common sense that we cannot design scintillator, OSL, and TSL materials by a basic solid state physics since the physics of whole processes of scintillation phenomena have not been fully established yet. In ionizing radiation induced luminescence phenomena, the problem to be solved is how a large number of secondary electrons dissipates their kinetic energy via an excited states interaction. My approach is to understand the phenomenon by simply assuming energy income and output, energy conservation. In near future, I would like to examine calorimeter like experiments to observe the thermal loss by the rise of temperature.

\section{Conclusion}


Scintillation, OSL, and TSL properties of famous scintillators, Ce-doped $\mathrm{CaF}_{2}$, Eu-doped $\mathrm{LiCaAlF}_{6}, \mathrm{Ce}-$ doped $\mathrm{LiCaAlF}_{6}$, and Pr-doped $\mathrm{LuAG}$ were investigated to study the relation between scintillation and storage-type emission. In Ce-doped $\mathrm{CaF}_{2}$, Eu-doped $\mathrm{LiCaAlF}_{6}$, and Pr-doped LuAG, scintillation and storage-type emission exhibited the inverse proportionality. In Ce-doped $\mathrm{LiCaAlF}_{6}$, the relation was a positive proportionality. More studies on various material systems are required to understand relation of ionizing radiation induced luminescence, scintillation and other storage-type emissions.

\section{Acknowledgements}

This work was mainly supported by a Grant in Aid for Scientific Research (A)-26249147 from the Ministry of Education, Culture, Sports, Science and Technology of the Japanese government (MEXT) and partially by JST A-step. Partial assistance from Nippon Sheet Glass Foundation for Materials Science and Engineering, Tokuyama Science foundation, Iketani Science and Technology Foundation, Hitachi Metals Materials Science Foundation, Mazda Foundation, JFE 21st century Foundation, and The Asahi Glass Foundation, the Cooperative Research Project of Research Institute of Electronics, Shizuoka University and Collaborative Research Program of Institute for Chemical Research, Kyoto University (2014-31) are also gratefully acknowledged.

\section{References}

[1] P. A. Rodnyi, Physical Processes in Inorganic Scintillators, CRC Press, Boca Raton, FL, (1997).

[2] G. Shani, Radiation Dosimetry: Instrumentation and Methods, CRC Press, Boca Raton, FL, (2000).

[3] T. Yanagida, Opt. Mater., 351987 (2013).

[4] Yukihara E. G., McKeever S. W. S., Optically Stimulated Luminescence: Fundamentals and Applications, Wiley (2011).

[5] Mckeever S. W.S. Thermoluminescence of Solids, Cambridge University Press (1985).

[6] T. Yanagida, Y. Fujimoto, K. Watanabe, K. Fukuda, N. Kawaguchi, Y. Miyamoto, H. Nanto, Rad. Meas., 71 162 (2014).

[7] N. V. Shiran, A. V. Gektin, S. V. Neicheva, E. P. Sysoeva, E. V. Sysoeva, K. Shimamura, A. Bensalah, T. Satonaga, Functional Materials 8, 732 (2001).

[8] T. Yanagida, N. Kawaguchi, Y. Fujimoto, K. Fukuda, Y. Yokota, A. Yamazaki, K. Watanabe, J. Pejchal, A. Uritani, T. Iguchi, A. Yoshikawa, Opt. Mater., 33, 1243-1247 (2011).

[9] M. Nikl, N. Solovieva, E. Mihokova, M. Dusek, A. Vedda, M. Martini, K. Shimamura, T. Fukuda phys. stat. sol. (a) 187, No. 1, R1-R3 (2001).

[10] T. Yanagida, A. Yoshikawa, Y. Yokota, S. Maeo, N. Kawaguchi, S. Ishizu, K. Fukuda, T. Suyama, Opt. Mater. 32 311-314 (2009).

[11] M. Nikl, H. Ogino, A.Krasnikov, A. Beitlerova, A.Yoshikawa, T. Fukuda, Phys. Stat. Sol. (a) 202 R4-R6 (2005).

[12] T. Yanagida, Y. Fujimoto, K. Kamada, D. Totsuka, H. Yagi, T. Yanagitani, Y. Futami, S. Yanagida, S. Kurosawa, Y. Yokota, A. Yoshikawa, M. Nikl, IEEE Trans. Nucl. Sci., 59 2146-2151(2012).

[13] N. Shiran, A. Gektin, S. Neicheva, V. Voronova, V. Kornienko, K. Shimamura, N. Ichinose, Radiat. Meas., 38 459-462 (2004).

[14] T. Yanagida, Y. Fujimoto, K. Watanabe, K. Fukuda, Rad. Meas., 71 148-152 (2014).

[15] T. Yanagida, K. Kamada, Y. Fujimoto, H. Yagi, T. Yanagitani, Opt. Mat.,35 2480-2485 (2013).

[16] T. Yanagida, Y. Fujimoto, T. Ito, K. Uchiyama, K. Mori, Appl. Phys. Exp., 7062401 (2014).

[17] T. Yanagida, Y. Fujimoto, H. Yagi, T. Yanagitani, Opt. Mater., 361044 (2014). 
[18] T. Yanagida, Y. Fujimoto, N. Kawaguchi, S. Yanagida, J. Ceram. Soc. Jpn., 121 989-991 (2013).

[19] M.V. Derdzyan, K.L.Ovanesyan, A.G.Petrosyan, A.Belsky, C.Dujardin, C.Pedrini, E. Auffray, P.Lecoq, M.Lucchini, K.Pauwels, J. Cryst. Growth, 361212 (2012).

[20] T. Yanagida, Y. Fujimoto, M. Koshimizu, K. Watanabe, H. Sato, H. Yagi, T. Yanagitani, Opt. Mater., 362016 (2014). 
Figure caption

Figure 1. X-ray induced radioluminescence spectra of Eu-doped $\mathrm{LiCaAlF}_{6}$ (left), Ce-doped $\mathrm{LiCaAlF}_{6}$ (center), and Pr-doped LuAG (right, selected samples).

Figure 2. X-ray induced scintillation decay time profiles of Eu-doped $\mathrm{LiCaAlF}_{6}$ (left), Ce-doped $\mathrm{LiCaAlF}_{6}$ (center), and Pr-doped LuAG (right, selected samples).

Figure 3. Pulse height spectra of Eu-doped $\mathrm{LiCaAlF}_{6}$ under ${ }^{252} \mathrm{Cf}$ neutron excitation (left), Ce-doped $\mathrm{LiCaAlF}_{6}$ under ${ }^{252} \mathrm{Cf}$ neutron excitation (center), and Pr-doped LuAG under ${ }^{137} \mathrm{Cs}$ g-ray excitation (right, selected samples).

Figure 4. OSL spectra of Eu-doped $\mathrm{LiCaAlF}_{6}$ under $470 \mathrm{~nm}$ stimulation (left), Ce-doped $\mathrm{LiCaAlF}_{6}$ under $405 \mathrm{~nm}$ stimulation (center), and Pr-doped LuAG under $405 \mathrm{~nm}$ stimulation (right, selected samples) after 10 Gy exposure. The inset of the panel of Ce-doped $\mathrm{LiCaAlF}_{6}$ shows the same plot for micro-pulling down made crystals.

Figure 5. TSL glow curves of Eu-doped $\mathrm{LiCaAlF}_{6}$ after 1 Gy exposure (left), Ce-doped $\mathrm{LiCaAlF}_{6}$ after 1 Gy exposure (center), and Pr-doped LuAG after 10 mGy exposure (right, selected samples).

Figure 6. The relation between the scintillation light yield (ph/5.5 MeV-a) and OSL intensity (photoluminescence quantum yield, \%) of Ce-doped $\mathrm{CaF}_{2}$. Data was selected from recent publication [6].

Figure 7. The relation between scintillation and OSL intensities of Eu-doped $\mathrm{LiCaAlF}_{6}$ under $470 \mathrm{~nm}$ stimulation (left), Ce-doped $\mathrm{LiCaAlF}_{6}$ under $405 \mathrm{~nm}$ stimulation (center), and Pr-doped LuAG under $405 \mathrm{~nm}$ stimulation (right, selected samples) after 10 Gy exposure.

Figure 8. The relation between scintillation and TSL intensities of Eu-doped LiCaAlF 6 after 1 Gy exposure (left), Ce-doped $\mathrm{LiCaAlF}_{6}$ after 1 Gy exposure (center), and Pr-doped LuAG after $10 \mathrm{mGy}$ exposure (right, selected samples). 
Figure1
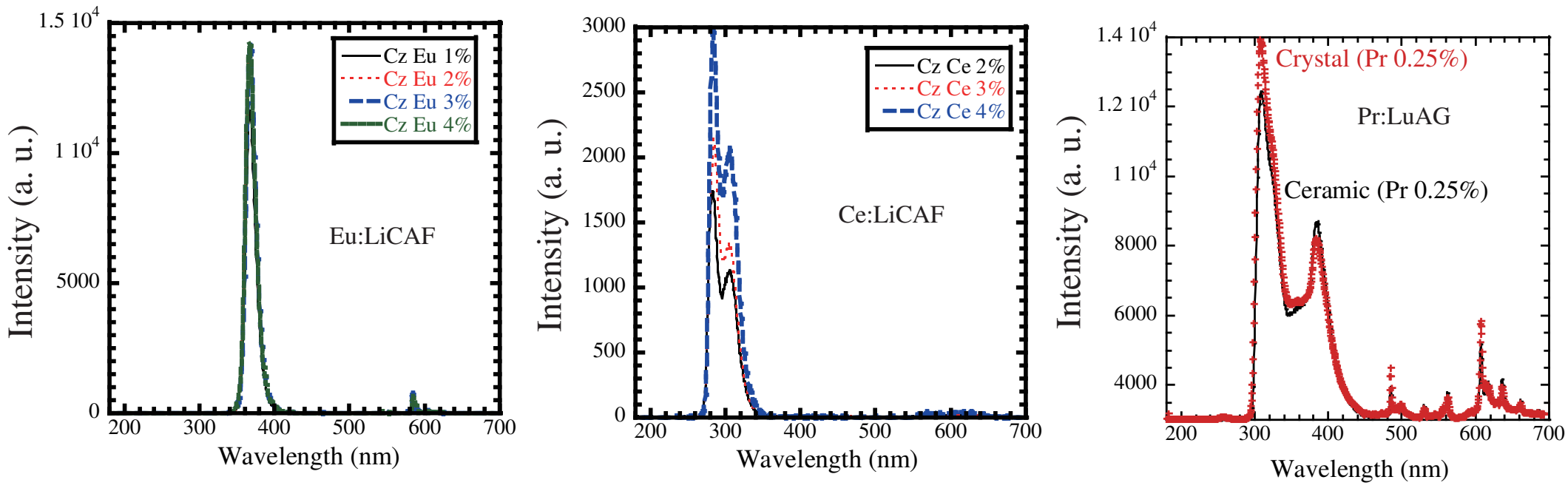
Figure2
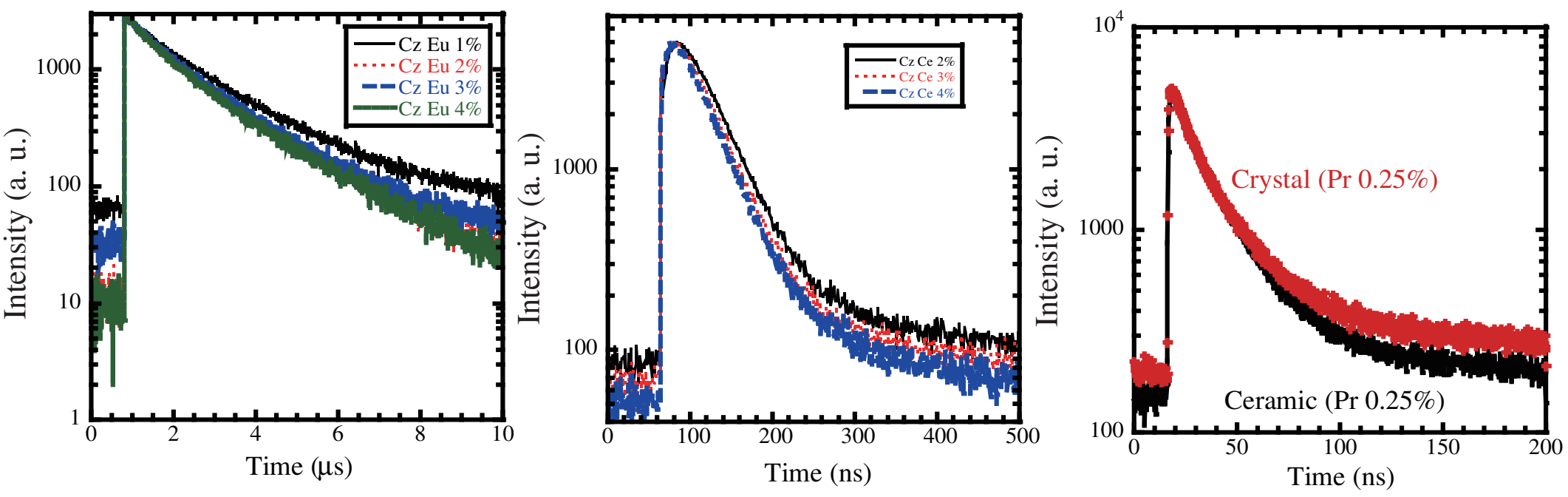
Figure3
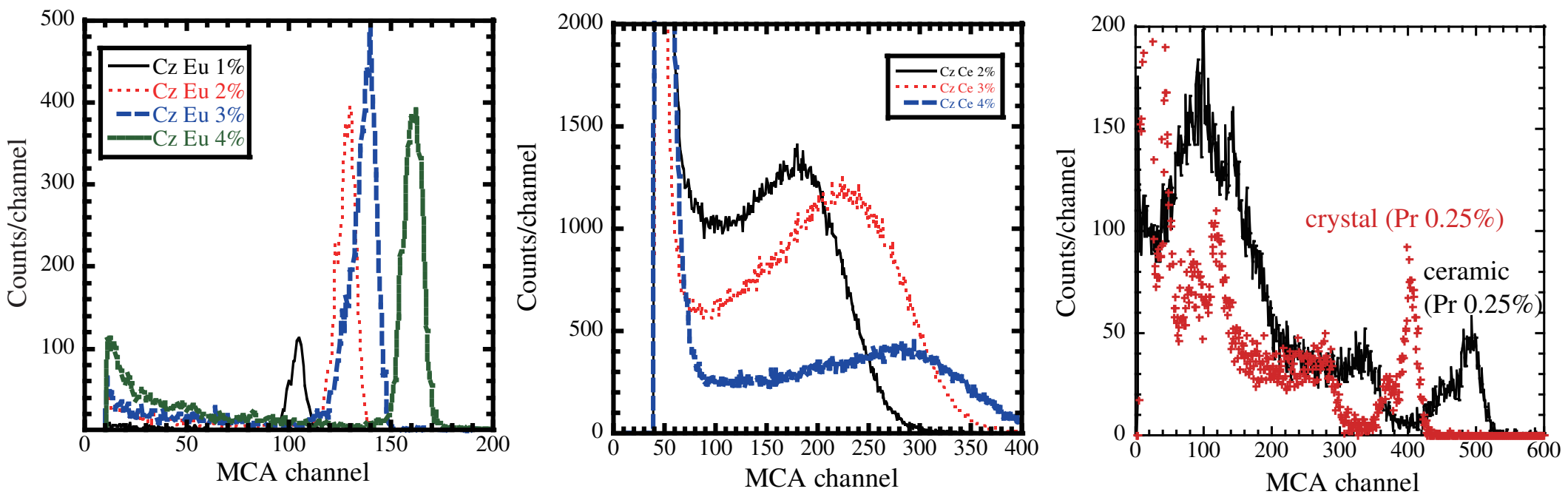
Figure4
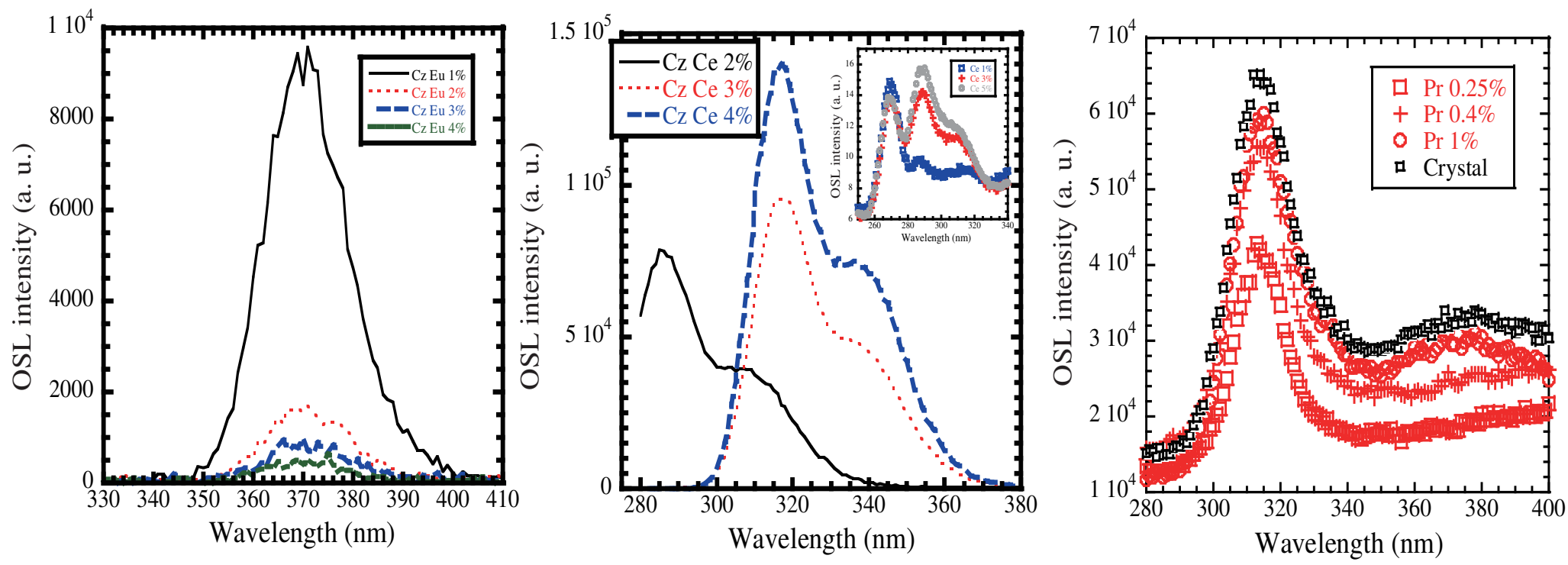
Figure6

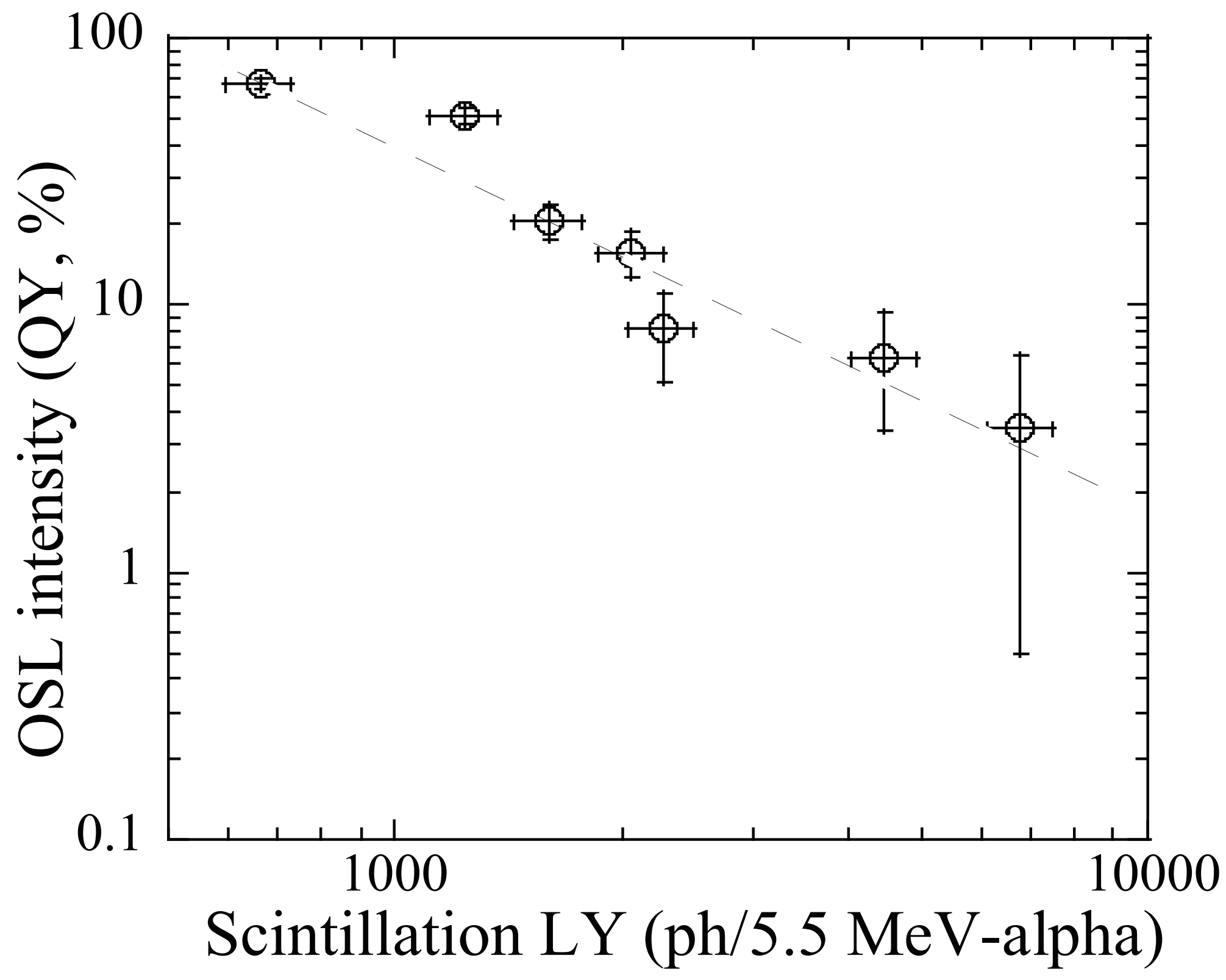


Figure8
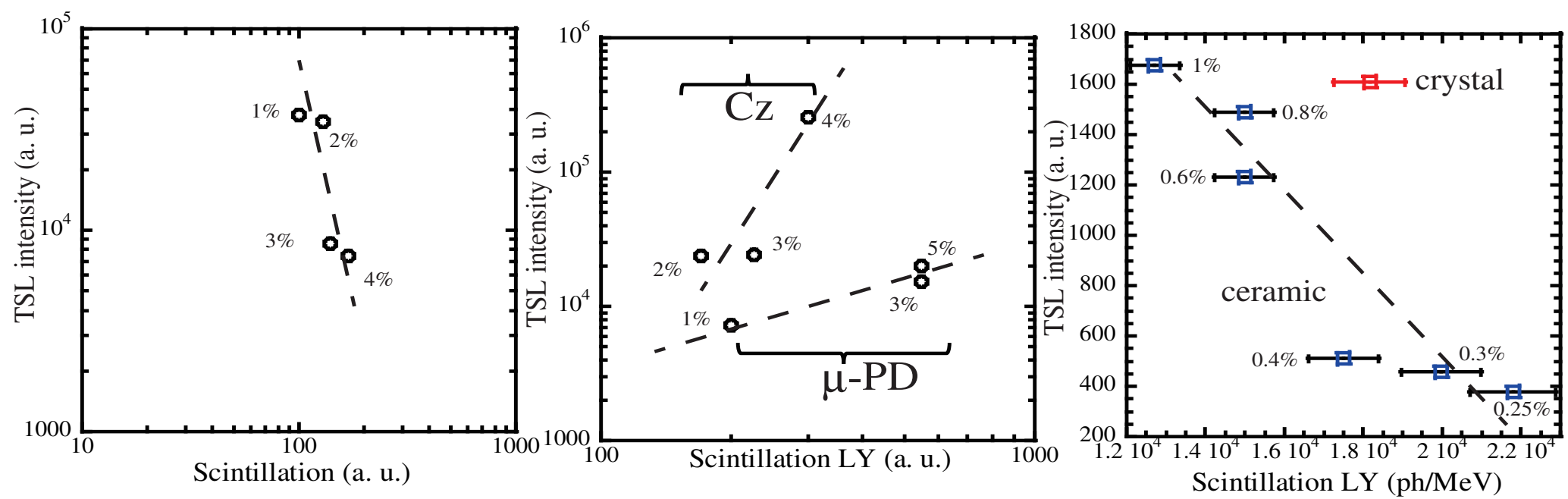